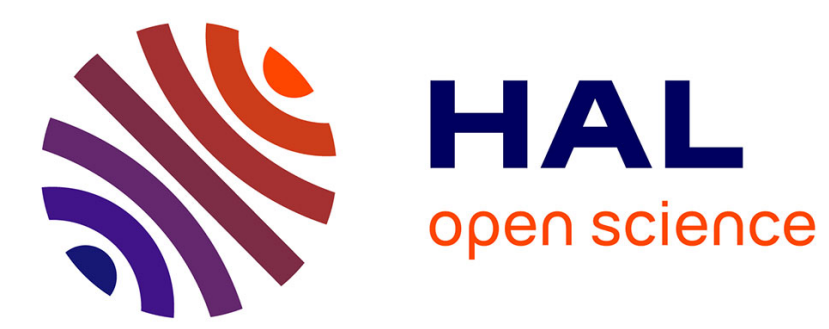

\title{
BRAGG-PHENOMENA IN THE FRESNEL REGION OF LIGHT DIFFRACTED BY ULTRASOUND
}

\author{
E. Blomme, O. Leroy
}

\section{To cite this version:}

E. Blomme, O. Leroy. BRAGG-PHENOMENA IN THE FRESNEL REGION OF LIGHT DIFFRACTED BY ULTRASOUND. Journal de Physique IV Proceedings, 1992, 02 (C1), pp.C1813-C1-816. 10.1051/jp4:19921178. jpa-00251139

\section{HAL Id: jpa-00251139 https://hal.science/jpa-00251139}

Submitted on 1 Jan 1992

HAL is a multi-disciplinary open access archive for the deposit and dissemination of scientific research documents, whether they are published or not. The documents may come from teaching and research institutions in France or abroad, or from public or private research centers.
L'archive ouverte pluridisciplinaire HAL, est destinée au dépôt et à la diffusion de documents scientifiques de niveau recherche, publiés ou non, émanant des établissements d'enseignement et de recherche français ou étrangers, des laboratoires publics ou privés. 


\title{
BRAGG-PHENOMENA IN THE FRESNEL REGION OF LIGHT DIFFRACTED BY ULTRASOUND
}

\author{
E. BLOMME and O. LEROY* \\ VH.T.I. Campus CATHO, Doorniksesteenweg 148, B-8500 Kortrijk, Belgium \\ *Interdisciplinar Research Center, K.U.L. Campus Kortrijk, E. Sabbelaan, B-8500 Kortrijk, Belgium
}

\begin{abstract}
Summary
The Fresnel region of light diffracted by a plane harmonic ultrasonic wave is examined under Bragg and near-Bragg conditions. For diffraction in the Bragg regime, the intensity of the diffracted light is sinusoidally modulated as a consequence of a two beam interference. In that case, the amplitude of the modulation does not depend on the plane of observation. For diffraction parameters in the near-Bragg regime, the light intensity modulation is produced by a multiple beam interference and its harmonic character disappears. In that case, the shape of the modulation also depends on the specific plane of observation.

\section{Sommaire}

La région de Fresnel de la lumière diffractée par une onde ultrasonore harmonique est étudiée pour des conditions de Bragg et presque Bragg. A 1 'incidence au voisinage de 1 'angle de Bragg et pour des fréquences ultrasonores suffisamment grand, I'intensité de la lumière diffractée est modulêe d'une façon sinusoidale comme l'onde ultrasonore elle-même, l'amplitude de la modulation étant indépendante du plan d'observation. D'autre part, pour des paramètres de diffraction se situant tout près du régime de Bragg, la modulation de la lumière diffractée n'est plus harmonique et la forme de la modulation dépend fortement du plan d'observation.
\end{abstract}

\section{GENERAL THEORY}

Let us consider a plane monochromatic and linearly polarized light wave of angular frequency $\omega=2 \pi v$ and wavelength $\lambda$ in vacuum, entering at an angle $\theta$ a rectangular, homogeneous, isotropic and transparant medium (e.g. a liquid column) with refractive index $\mu_{0}$. The medium is disturbed by a plane ultrasonic harmonic wave of frequency $\omega^{\star}=2 \pi \nu^{\star}$ and wave number $\mathrm{k}^{\star}=2 \pi / \lambda^{\star}$, propagating in the X-direction (Fig. 1 ). After leaving the ultrasonically disturbed medium, the light intensity in the Fresnel zone of the diffracted light is modulated in both time and space. Assuming that the intensity of the incident light is equal to 1 , the light intensity distribution in a plane parallel to the exit plane $z=L$ is described by the Fourier series [1]

$$
I(x, z, t)=\sum_{m=-\infty}^{+\infty} C_{m}(z) \exp \left[-i m\left(\omega^{\star} t-k^{\star} x\right)\right]
$$

with coefficients

$$
C_{m}(Z)=\exp [2 i m(m-\beta) \pi z] \sum_{p=-\infty}^{+\infty} \phi_{m+p}(L) \overline{\phi_{p}(L)} \exp (4 i m p \pi z)
$$


$Z$ is a normalized spatial variable defined by

$$
z=\frac{z-L}{\bar{D}},
$$

$L$ being the effective width of the sound beam, and $D$ the distance

$$
D=\frac{2 \mu_{0} \lambda^{\star 2}}{\lambda}
$$

Next, $\theta=\theta / \theta_{\mathrm{B}}$ expresses the angle of incidence in units of the Bragg angle $\theta_{\mathrm{B}}=\operatorname{ArC}\left(\lambda / 2 \mu_{\mathrm{O}} \lambda^{\star}\right)$. Finally, $\phi_{\mathrm{p}}(\mathrm{L})$ represents the amplitude of the diffracted light component with frequency $\omega+p w^{\star}$ evaluated in the plane $z=L$ ( $i . e . \quad z=0$ ) and the bar denotes the complex conjugate. The amplitude $\phi_{\mathrm{p}}(z)$ can be obtained as a solution of the Raman-Nath system

$$
2 L \frac{d \phi_{n}}{d z}-v\left[\phi_{n-1}-\phi_{n+1}\right]=4 n \pi i(n-\beta) \frac{L}{D} \phi_{n}, \quad n=0, \pm 1, \pm 2, \ldots
$$
subject to the boundary condition $\phi_{n}(0)=\delta_{n, 0}[1] . v$ is the familiar
Raman-Nath parameter

$$
\mathrm{v}=\frac{2 \pi \mu_{1} \mathrm{~L}}{\lambda \cos \theta}
$$

$\mu_{1}$ representing the maximum variation of the refractive index. Expressions (1) and (2) are applicable to any diffraction regime. After calculation of the suitable amplitudes $\phi_{p}(\mathrm{~L})$, the light intensity distribution in the Fresnel region of the diffracted light is obtained from (1) and (2). Previous papers already dealt with the Raman-Nath regime [ 1,2] and the intermediate diffraction regime [3] . In the next sections, the Bragg and the near-Bragg regime are treated.

\section{THE BRAGG REGIME}

In the Bragg regime, all diffraction orders disappear, except the zero and first order (Fig. la). Hence, putting $\phi_{n}=0$ if $n \neq\{0,1\}$, the infinite system (5) is reduced to a set of 2 equations. For angles in the neighbourhood of the Bragg angle, the solution of this system is given by [4]

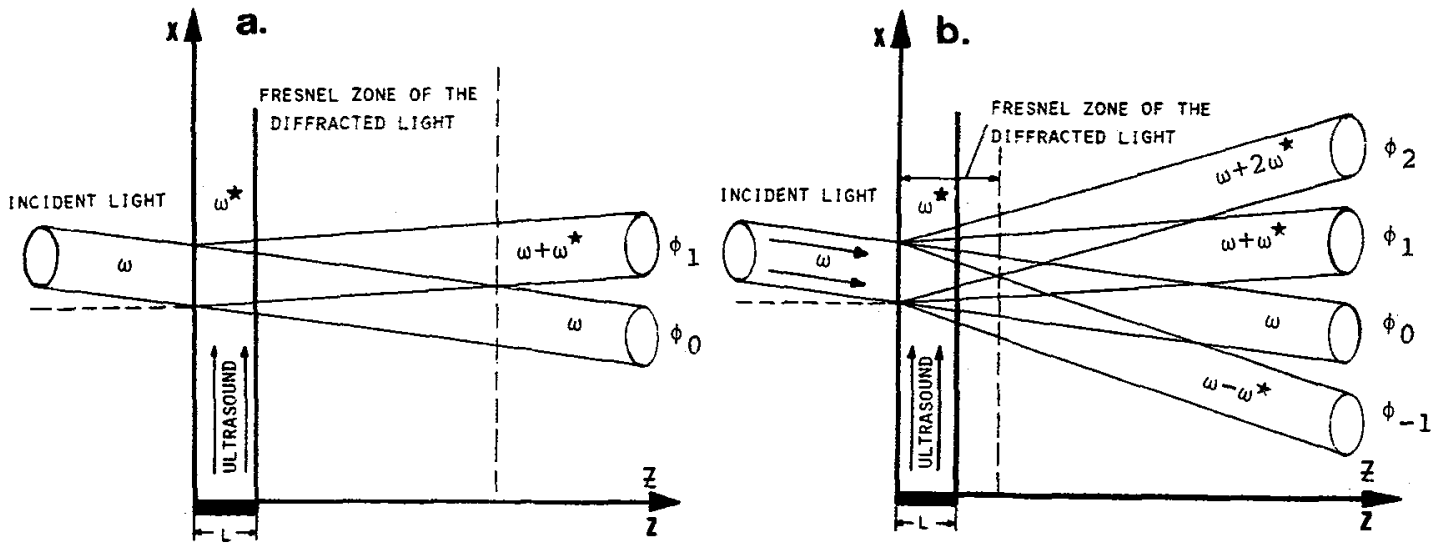

Fig. 1. Ultrasonic light diffraction in (a) the Bragg regime and (b) the near-Bragg regime: geometrical representation, diffraction orders and their frequencies. 


$$
\begin{aligned}
& \phi_{0}(L)=\exp \left(\frac{1}{4} i Q \Delta \beta\right)\left[\cos \gamma-\frac{i Q \Delta \beta}{4 \gamma} \sin \gamma\right] \\
& \phi_{1}(L)=\exp \left(\frac{1}{4} i Q \Delta \beta\right) \frac{v \sin \gamma}{2 \gamma}
\end{aligned}
$$

where

$$
\gamma=\frac{1}{4} \sqrt{Q^{2}(\Delta B)^{2}+4 v^{2}} \text { and } Q=\frac{2 \pi \lambda L}{\mu_{0} \lambda^{\star^{2}}}
$$

$Q$ being the Klein-Cook parameter; $\Delta \beta=1-\beta$ represents the deviation of the actual angle of incidence from the Bragg angle in units of the latter. At exact Bragg incidence $(\Delta \beta=0)$, expressions (7) and ( 8 ) reduce to the well-known formulae

$$
\phi_{0}(L)=\cos \frac{1}{2} \mathrm{v}, \phi_{1}(L)=\sin \frac{1}{2} \mathrm{v} .
$$

Introducing (7) and (8) into the Fourier coefficients (2), the following expression for the intensity distribution in the Fresnel zone of the diffracted light is obtained:

$$
I(x, z, t)=1+\frac{V \sin 2 \gamma}{2 \gamma} \cos \left(\omega^{\star} t-k^{\star} x-2 \pi \Delta \beta Z\right)+\frac{v Q \Delta \beta}{4 \gamma^{2}} \sin ^{2} \gamma \sin \left(\omega^{\star} t-k^{\star} x-2 \pi \Delta B Z\right) .
$$

At pure Bragg incidence, the 2 nd component vanishes and we obtain the simple expression

$$
I(x, z, t)=1+\operatorname{sinv} \cdot \sin \left(\omega^{\star} t-k^{\star} x+\frac{\pi}{2}-2 \pi \Delta \beta Z\right) \text {. }
$$

In general, if $\Delta \beta \neq 0$, both harmonic components are present in (11), and as the addition of two harmonic waves of the same frequency again is harmonic, (11) can be written in the more compact form

$$
I(x, z, t)=A \sin \left(\omega^{\star} t-k^{\star} x+\psi\right)
$$

with amplitude and initial phase

$$
A=\frac{v|\sin \gamma|}{\gamma} \sqrt{\cos ^{2} \gamma+\frac{Q^{2}(\Delta \beta)^{2}}{16 \gamma^{2}} \sin ^{2} \gamma}
$$
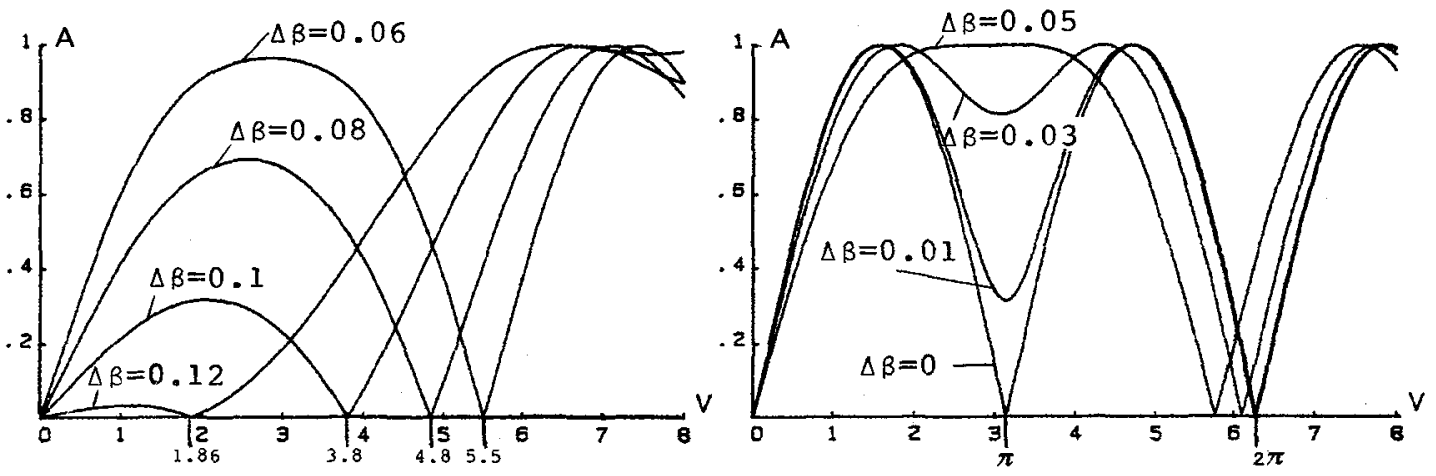

Fig. 2. Diffracted light amplitude as a function of the Raman-Nath parameter for $Q=100$ and for a series of angles close to the Bragg angle. The indicated zeros in the $\mathrm{f} / \mathrm{g}$. on the left are calculated from (16). 

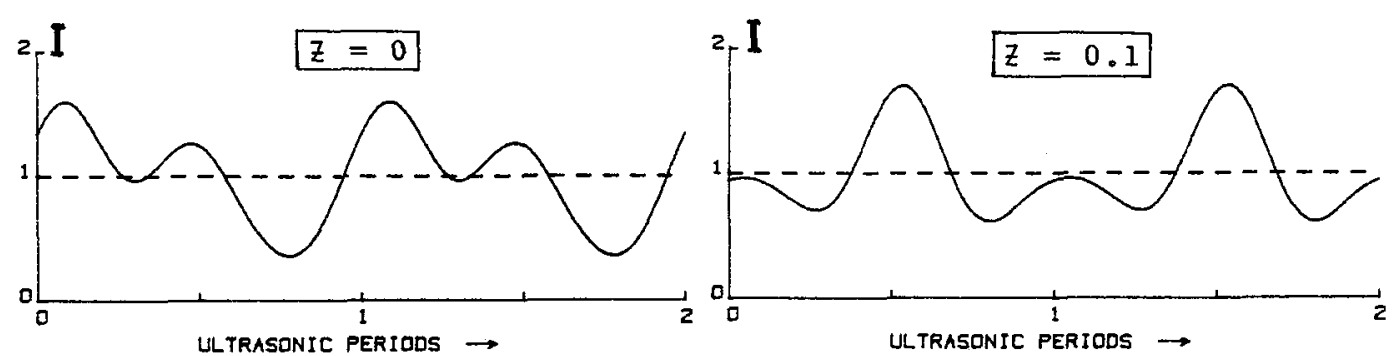

Fig. 3. Diffracted light intensity signal over 2 ultrasonic time periods in two different planes of observation at Bragg incidence of the light and for $v=\pi$ and $Q=10$. In this case, $3 \%$ of the light is diffracted in each of the additional diffraction orders $\phi_{-1}$ and $\phi_{2}$.

$$
\psi=\operatorname{Arctan}\left[\frac{4 \gamma}{Q \Delta \beta} \cot \left(\frac{1}{4} \Omega \Delta \beta\right)\right]-2 \pi \Delta \beta Z+\varepsilon \pi
$$

with $\varepsilon=0$ if $\Delta \beta>0$ and $\varepsilon=1$ if $\Delta \beta<0$.

From this analysis, the following conclusions are obvious:

- The diffracted light intensity signal has a harmonic character.

- The amplitude of the light intensity signal is independent of the plane of observation.

- At exact Bragg incidence, the modulation disappears if $v=\ell \pi(\ell \in N)$. In that case, only the zero or the first order are present in the diffraction spectrum.

- For any angle of incidence in the neighbourhood of the Bragg angle, there are values of $v$ at which the modulation vanishes (Fig. 2).

Indeed, $A=0$ if and only if $\sin \gamma=0$. In view of (9), this condition is fulfilled for values of $v$ satisfying the relation

$$
v=\frac{1}{2} \sqrt{16 p^{2} \pi^{2}-Q^{2}(\Delta \beta)^{2}}
$$

where $p$ is any integer such that the square root is real. In fact, for these values of $\mathrm{v}$, the first diffraction order vanishes.

- Conversely, for a fixed value of $v$, there are angles of incidence at which the modulation vanishes (see also Nomoto [5]).

\section{THE NEAR-BRAGG REGIME}

For parameters in the near-Bragg regime, two additional orders appear, one at each side of the central Bragg orders (Fig. 1b). In that case, the complex solution of system (5), now reduced to 4 equations, still can be solved analytically [ 6] . Performing the substitution of $\phi_{-1}, \phi_{0}$, $\phi_{1}$ and $\phi_{2}$ into (2), the following conclusions can be drawn:

- The harmonic character of the light intensity modulation is affected. - Not only the phase of the light intensity signal, but the whole modulation profile depends on the plane of observation.

Both properties are illustrated on Fig. 3.

\section{References}

[1] BLOMME, E. and LEROY, O., J. Acoust. SOC. Am., to be published.

[2] COOK,B.D., J. Acoust. Soc. Am., 60 (1976) 95-99.

[3] BLOMME, E., KWIEK, P., LEROY,O and REIBOLD, R., Acustica, 73 (1991) $134-143$.

[4] PHARISEAU, P., Proc. Indian Acad. Sci., 44 (1956) 165-170.

[ 5] NOMOTO,O., J. Acoust. SOC. Jpn., 3 (1982) 151-165.

[6] BLOMME, E. and LEROY,O., Acustica, 59 (1986) 182-192. 